\title{
Electrical stimulation therapy of the lower esophageal sphincter is successful in treating GERD: long-term 3-year results
}

\author{
Leonardo Rodríguez ${ }^{1}$ Patricia A. Rodriguez ${ }^{1} \cdot$ Beatrice Gómez $^{1}$. \\ Manoel Galvao Netto ${ }^{2} \cdot$ Michael D. Crowell ${ }^{3}$ Edy Soffer $^{4}$
}

Received: 9 July 2015/Accepted: 1 September 2015/Published online: 20 October 2015

(C) The Author(s) 2015. This article is published with open access at Springerlink.com

\begin{abstract}
Background Electrical stimulation of the lower esophageal sphincter (LES) has been shown to improve outcomes in patients with gastroesophageal reflux disease (GERD) at 2 years. The aim of the study was to evaluate the safety and efficacy of LES stimulation in the same cohort at 3 years. Methods GERD patients with partial response to PPI, with $\%$ 24-h esophageal $\mathrm{pH}<4.0$ for $>5 \%$, with hiatal hernia $<3 \mathrm{~cm}$ and with esophagitis $\leq$ LA grade $\mathrm{C}$ were treated with LES stimulation in an open-label 2-year trial. All patients were on fixed stimulation parameter of $20 \mathrm{~Hz}$, $220 \mu \mathrm{s}, 5 \mathrm{~mA}$ delivered in twelve, 30-min sessions. After completing the 2-year open-label study, they were offered enrollment into a multicenter registry trial and were evaluated using GERD-HRQL, symptom diaries and $\mathrm{pH}$ testing at their 3-year follow-up.

Results Fifteen patients completed their 3-year evaluation [mean (SD) age $=56.1$ (9.7) years; men $=8$ ] on LES stimulation. At 3 years, there was a significant improvement in their median (IQR) GERD-HRQL on electrical stimulation compared to both their on PPI [9 (6-10) vs. $1(0-2)$,
\end{abstract}

Presented at the SAGES 2015 Annual Meeting, April 15-18, 2015, Nashville, Tennessee.

Edy Soffer

esoffer@usc.edu

1 Centro Clinico de Obesidad Diabetes y Reflujo, Santiago, Chile

2 Department of Surgery, Gastro Obeso Center, Sao Paulo, Brazil

3 Department of Gastroenterology, Mayo Clinic, Scottsdale, AZ, USA

4 Department of Medicine, Keck School of Medicine at the University of Southern California, Los Angeles, CA, USA $p=0.001]$ and off PPI [22 (21-24) vs. $1(0-2), p<0.001]$. Median 24-h distal esophageal acid exposure was significantly reduced from $[10.3(7.5-11.6) \%$ at baseline vs. 3 $(1.9-4.5) \%, p<0.001]$ at 3 years. Seventy-three \% (11/15) patients had normalized their distal esophageal acid exposure at 3 years. Remaining four patients had improved their distal esophageal acid exposure by $39-48 \%$ from baseline. All but four patients reported cessation of regular PPI use ( $>50 \%$ of days with PPI use); three had normal esophageal $\mathrm{pH}$ at 3 years. There were no unanticipated device- or stimulation-related adverse events or untoward sensation reported during the 2- to 3-year follow-up.

Conclusion LES-EST is safe and effective for treating patients with GERD over long-term, 3-year duration. There was a significant and sustained improvement in esophageal acid exposure and reduction in GERD symptoms and PPI use. Further, no new GI side effects or adverse events were reported.

Keywords GERD - Treatment - Electrical stimulation Lower esophageal sphincter - Esophageal acid · Outcomes

Gastroesophageal reflux disease (GERD) is a chronic disease with high global prevalence of up to $20 \%$ [1]. GERD occurs when a weak or dysfunctional lower esophageal sphincter (LES) exposes the esophagus to acidic stomach contents, resulting in bothersome symptoms of heartburn, regurgitation, and chest pain and a significant impairment of patients' quality of life [2]. Acid damage can lead to stricture formation and Barrett esophagus, which can eventually lead to esophageal adenocarcinoma, one of the fastest rising cancers in the Western world [3]. Excessive esophageal acid exposure is the hallmark of this disease, and measurement of 24- to 48-h esophageal acid exposure is the most robust gold-standard for the diagnosis of GERD [4]. 
Current therapies aim to treat GERD by controlling or eliminating esophageal acid exposure. Medications such as proton pump inhibitors (PPI) minimize esophageal acid exposure by blocking gastric acid secretion; however, due to persistent LES dysfunction, reflux of non-acidic gastric contents continues at comparable magnitude, resulting in persistent symptoms in up to $40 \%$ of patients [5]. Laparoscopic fundoplication for sphincter augmentation controls reflux of all gastric contents; however, the anatomical alteration at the level of the GE junction is associated with multiple side effects, some debilitating, that plague the procedure [6], as well as recurrence of symptoms in a sizable number of patients $[6,7]$. Concerns about optimal outcomes in low-volume centers persist $[8$, 9]. Perhaps as a result, the number of fundoplication procedures performed has been declining [10].

An ideal antireflux procedure should result in longterm control of esophageal acid exposure, without any significant side effects or adverse events, thus improving patients' symptoms and quality of life without dependence on daily medications [11]. We had previously reported the results from our long-term, 2-year open-label trial of LES stimulation in patient of GERD [12]. The current report describes the results of stimulation therapy after extension of the follow-up in the same cohort of patients to 3 years.

\section{Materials and methods}

A Web-based international multicenter registry has been established by EndoStim (The Hague, The Netherlands), the manufacturer of the LES stimulation system, to allow physicians to track the outcomes of their patients treated with LES stimulation in their clinical practice outside of clinical trials (NCT02441400). GERD patients with partial response to PPI, with hiatal hernia $\leq 3 \mathrm{~cm}$ and with $\leq$ grade $\mathrm{C}$ esophagitis were enrolled in a single-center, open-label trial of electrical stimulation of the LES using the EndoStim ${ }^{\circledR}$ LES stimulation system (Fig. 1). The system is implanted laparoscopically and is programmed to deliver intermittent stimulation to the LES. The details of the open-label trial and the 2-year results have been previously reported [12]. At the end of the 2-year follow-up, patients were offered to participate in a registry trial to allow for a follow-up of up to 5 years, to provide an assessment of long-term clinical outcomes. The registry trial was approved by the Institutional Review Board of Clinica Indisa, Santiago, Chile. At their 3-year follow-up, patients who enrolled in the registry trial were offered evaluation using GERD-HRQL, and daily diary questionnaires, SF-12, as well as esophageal $\mathrm{pH}$ testing to objectively measure their esophageal acid exposure.
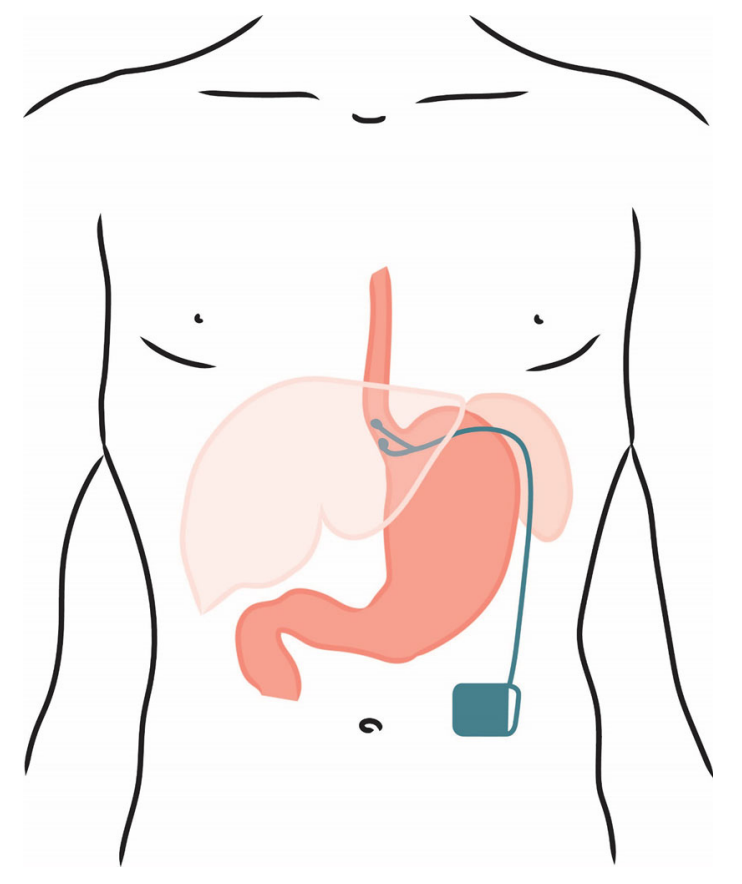

Fig. 1 LES stimulation system with electrodes implanted in the LES and the pulse generator implanted in a subcutaneous pocket in the anterior abdominal wall

The effect of electrical stimulation of the LES on patient symptoms was measured using GERD-HRQL (on PPI and 2 weeks off PPI at baseline and after 2 weeks off PPI during follow-up), symptoms and medication use reported in a daily diary, and general quality of life measured using SF-12 (on PPI and 2 weeks off PPI at baseline and after 2 weeks off PPI during follow-up visits) using related samples Wilcoxon signed-rank test was used for comparison. Change in $\%$ time distal esophageal $\mathrm{pH}$ was $<4.0$ was assessed by comparing the results at baseline to the 3-year follow-up. LES electrical stimulation therapy (EST) was evaluated using related samples Wilcoxon signed-rank test. A $p$ value of $<0.05$ was considered statistically significant.

\section{Results}

Eighteen patients consented to enroll in the registry trial, and 15 of those patients were available for evaluation at 3 years. The details of patient follow-up are presented in Fig. 2, and the baseline characteristics of patients that underwent 3-year evaluation are provided in Table 1.

There were no additional adverse events reported between years 2 and 3 of follow-up. The details of adverse events prior to 2 years have been previously reported [13] and include two serious adverse events: acute non-cardiac retrosternal chest pain and surgery for solitary thyroid nodule, both deemed not related to device or procedure by 
Fig. 2 Patient follow-up chart

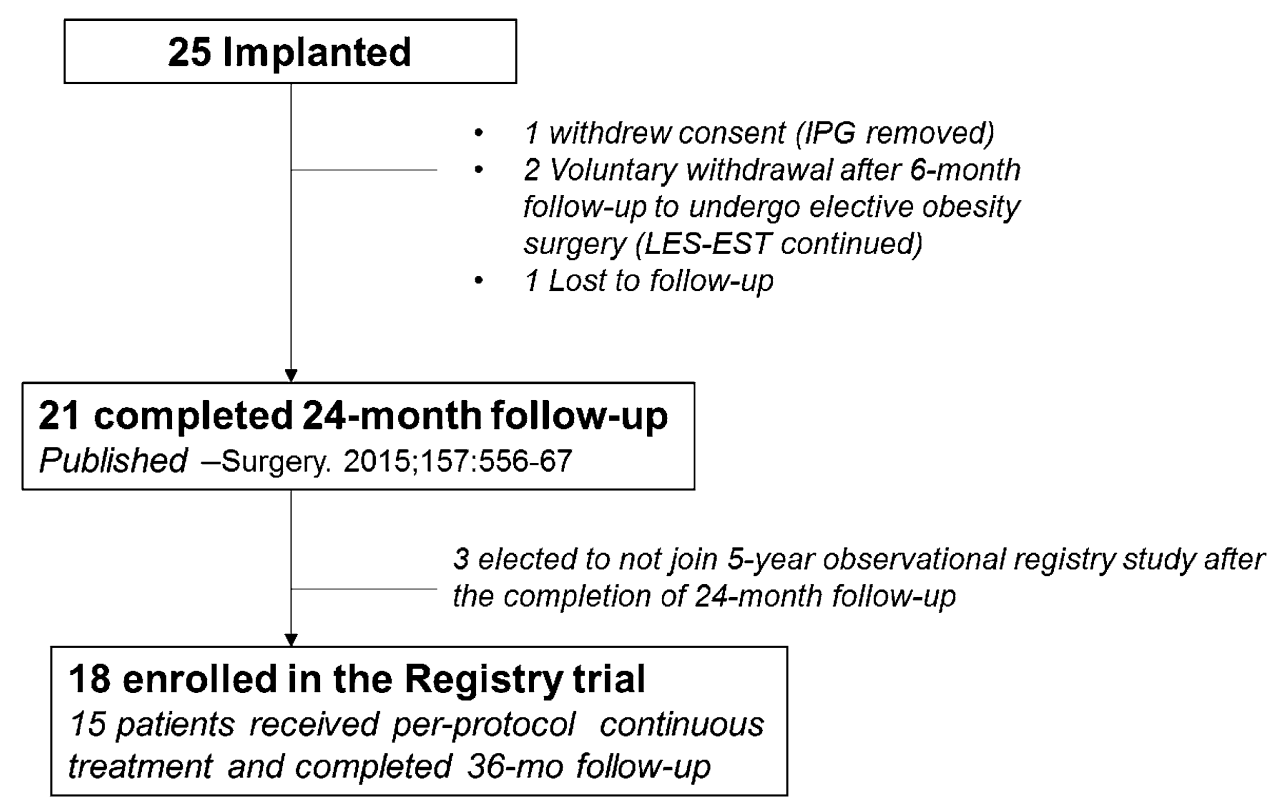

an independent data safety and monitoring board. There were a total of 63 non-serious adverse events in the trial; 12 were classified as device/procedure related. Most device-/ procedure-related events were typical of a surgical implant procedure (e.g., implant site pain, post-op nausea), mildmoderate in severity and resolved with or without intervention. There were no stimulation-related side effects or sensation, and no events of dysphagia were reported.

The median distal esophageal acid exposure was significantly improved at 3 years at $3 \%$ (IQR 1.9-4.5) compared to $10.1 \%(7.8-13 ; p<0.001)$ in the whole cohort and $10.3 \%(4.0-7.8 ; p<0.001)$ in the matched cohort at baseline (Fig. 3). Seventy-three \% (11/15) reported normalization $(<4.0 \%$ of $24 \mathrm{~h})$ of their distal esophageal acid exposure. The remaining four patients had 39-48\% improvement in their distal esophageal acid exposures. The median DeMeester score was significantly improved at 3 years from $36.9(30.8-44.3)$ at baseline to 12.8 (7.2-18.8; $p=0.0003$ ).

Three patients underwent blinded turn-off of LES-EST after their 18-month follow-up, and one patient had her therapy accidentally turned-off by inadvertent use of a magnet therapy for her arthritis at month 15. All four patients demonstrated worsening of their distal esophageal acid exposure at 2 years compared to their on-therapy at 12 months, though values improved, they did not return to their baseline esophageal acid exposure even after $>3$ months of cessation of LES-EST [12]. All these patients had their stimulation turned back after their 2-year visit, and three of these patients underwent esophageal $\mathrm{pH}$ testing at 3 years. All three patients had significantly improved or normalized their distal esophageal acid exposure, suggesting a causal association between improvement in esophageal acid exposure and LES stimulation (Fig. 4).

The median composite GERD-HRQL scores were significantly improved at 3 years at 1.0 (IQR 0-2.0) compared to $23.5(21-25 ; p<0.001)$ off PPI and 9.0 $(6-10 ; p<0.001)$ on PPI, respectively, in the whole cohort and 22 (21-24; $p<0.001)$ off PPI and 9.0 (6-10; $p<0.001)$ on PPI, respectively, in the matched cohort at baseline (Fig. 5). All patients reported clinical significance ( $>50 \%$ improvement) in their composite GERDHRQL score versus both their baseline off-PPI scores and on-PPI scores. The SF-12 scores for both physical and mental health improved numerically compared with baseline on- and off-PPI scores, but did not reach statistical significance. There was a significant reduction in dependence on PPI medications. All patients at baseline were on either single- or double-dose PPI for a median duration of 6.0 years. Seventy-three $\%$ of patients were free of PPI dependence at their 3-year follow-up (Fig. 6). Three patients were using daily PPI, two had normal distal esophageal acid exposure, and one was improved by $39 \%$, while one patients was using PPI 4X/week and had normal distal esophageal acid exposure. All esophageal pH measurements were off PPI.

All patients in the cohort had erosive esophagitis at baseline. Twelve patients underwent endoscopy at their 3-year follow-up with $50 \%$ (6/12) patients showing improvement in their esophagitis by $\geq 1$ grade, and $25 \%$ (3/12) patients each had stable grade A and worsening 
Table 1 Baseline characteristics and relevant medical/GERD history of patient treated per-protocol with LES stimulation and completing their 3-year evaluation

\begin{tabular}{lll}
\hline Characteristic & $N$ & Mean (SD) \\
\hline Age (years) & 15 & $56.1(9.7)$ \\
Body mass index (BMI) & 15 & $27.4(3.2)$ \\
Gender & & \\
Male & 7 & \\
Female & 3 & \\
BMI class & 9 & \\
Normal (<25) & 3 & \\
Overweight ( $\geq 25$ and $<30)$ & $100 \%$ \\
Obese ( $\geq 30)$ & \\
\hline Patients using daily PPI & $>12.2(9.1)$ years \\
Duration of GERD symptoms & $>10(7.5-12.5)$ years \\
Mean (SD) & \\
Median (quartile) & $>5.9(3.3)$ years \\
Duration of PPI use & \\
Mean (SD) &
\end{tabular}

$S D=$ standard deviation, $I Q R$ interquartile range

(grade A to grade B) esophagitis. Two of the 3 patients that had worsening esophagitis and all 3 patients with stable esophagitis had normal esophageal acid exposure at their 3-year evaluation. None of the patients had developed Barrett esophagus at their 3-year follow-up on LES stimulation therapy.

\section{Discussion}

This is the first report of sustained control of esophageal acid exposure at 3 years with LES electrical stimulation in patients with GERD, who were at least partially responsive to PPI at baseline evaluation. Along with objective improvement in GERD, there was sustained improvement in GERD symptoms and GERD-related quality of life, and elimination or reduction in need for daily PPI medications, without any new adverse events.

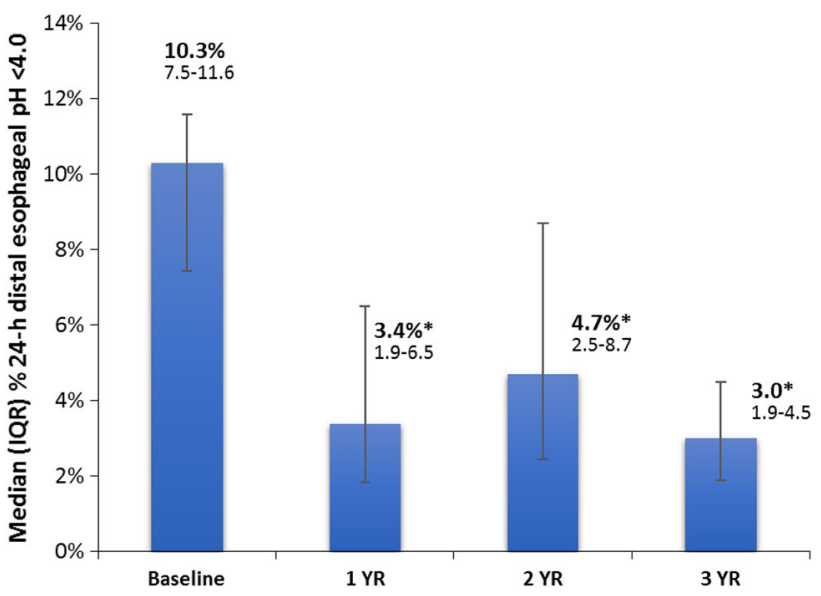

Fig. 3 Sustained improvement in the distal esophageal acid exposure on LES stimulation at 3-year follow-up. Data: median, IQR. $73 \%$ reported normalization $(<4.0 \%$ of $24 \mathrm{~h})$ in their distal esophageal acid exposure at their 3-year follow-up

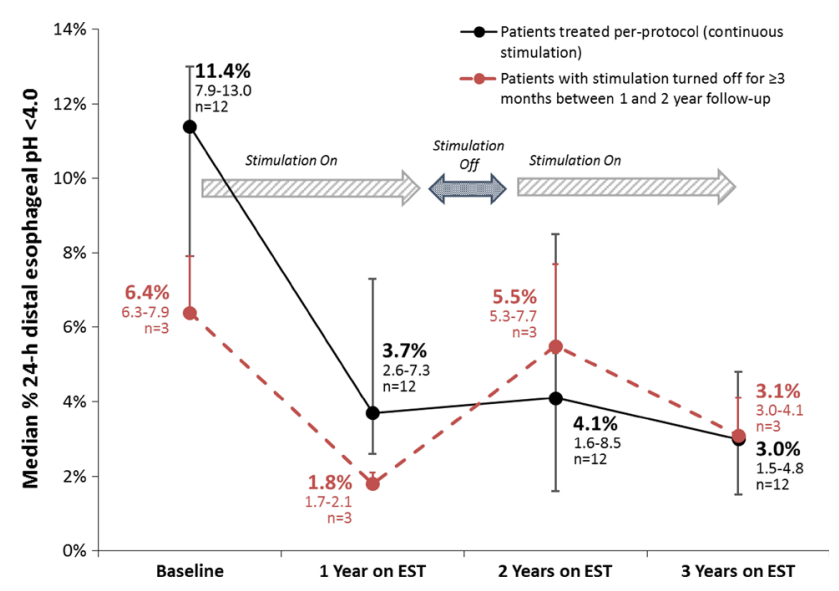

Fig. 4 Effect of blinded turn-off and turn-on on esophageal acid exposure. Esophageal acid exposure increased on blinded turn-off before the 2-year $\mathrm{pH}$ study and then improved after blinded turn-on at 2 years, as measured at their 3-year follow-up. EST electrical stimulation therapy

Excessive esophageal acid exposure due to the failure of the antireflux barrier is the hallmark of GERD. The current gold-standard objective test to diagnose GERD is the prolonged ambulatory esophageal pH-metry [14]. The total time with esophageal $\mathrm{pH}<4$ as recorded by a probe placed $5 \mathrm{~cm}$ above the LES and the composite JohnsonDeMeester score have the highest sensitivity and specificity in the diagnosis of GERD [4]. Control of esophageal acid exposure is the most robust measure of effectiveness of a GERD therapy, although $\mathrm{pH}$ testing has not been an endpoint for most GERD therapy clinical trials. Our results show a significant and sustained improvement in esophageal acid exposure and Johnson-DeMeester composite scores with LES stimulation at 3-year follow-up. Almost 


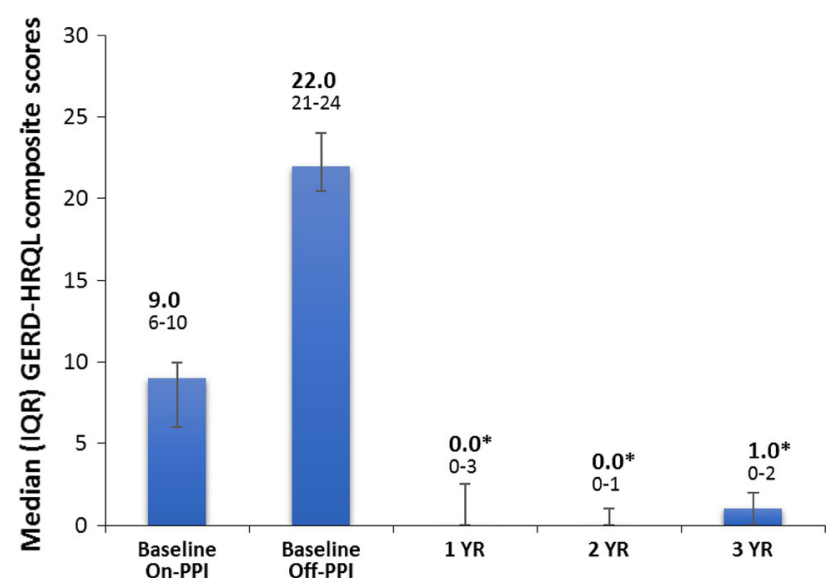

Fig. 5 Sustained improvement in GERD symptoms as measured by the composite GERD-HRQL scores at 3-year follow-up. Data: median, IQR. All patients reported clinically significant improvement ( $\geq 50 \%$ improvement in the composite GERD-HRQL score) in symptoms at 3 years compared to baseline off PPI and better composite GERD-HRQL scores than baseline on PPI

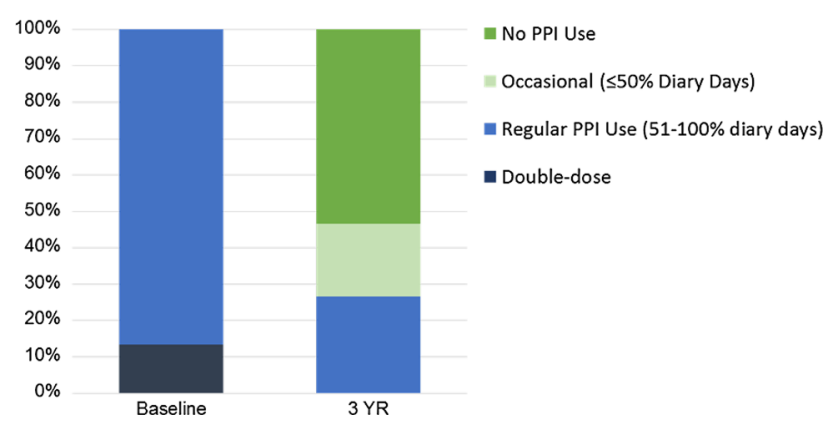

Fig. 6 PPI medication use at baseline and at 3-year follow-up. Most patients $(73 \%)$ were free from PPI dependence (dependence defined as $\geq 50 \%$ diary days with PPI use)

3/4th of the patients, mostly partial responders, evaluated at 3-year follow-up on LES stimulation therapy had normalized their esophageal acid exposure, while the remaining 1/4th had improved (39-48 \%) but not normalized esophageal acid exposure. This important observation highlights the durability of acid exposure control with LES stimulation over time. Sustained long-term control of esophageal acid exposure is essential for any effective GERD therapy. This contrasts with the abnormal esophageal $\mathrm{pH}$ values documented in $56 \%$ of patients with refractory heartburn on twice daily PPI, and in $30 \%$ of patients with typical GERD symptoms who were well controlled on once daily PPI $[15,16]$. In another study, $50 \%$ of patients with GERD, who achieved complete symptom control with PPI therapy, were found to have abnormal esophageal exposure while on PPI [17].

In addition to the objective improvement in esophageal acid exposure, there was also a significant and sustained improvement in patient-centric outcomes of GERD symptoms, both on and off PPI as well as acid suppression medication use at 3 years on LES stimulation. Almost twothirds of the patients chose to undergo antireflux surgery due to incomplete symptom control with medication, and the rest were unwilling to take medications due to quality of life, safety or cost concerns. The proportion of patients and physicians concerned about long-term safety of highdose PPI, and the literature on this topic is growing, resulting in an increasing number of patients and physicians who desire an alternative to long-term PPI [18]. Dependence on daily PPI was eliminated in $80 \%$ with additional $20 \%$ using PPI, albeit at a reduced dose. Although elimination of any PPI use in all patients is the most desirable outcome, a significant reduction in the cumulative dose is also quite beneficial, as some observational studies showed that the adverse effect profile of PPI is dose dependent [19].

Finally, the greatest advantage of electrical stimulation is its safety profile, observed in other applications over the years. Except for anticipated events typically seen after a laparoscopic implant procedure, no other significant adverse events have been reported in our patients. More importantly, due to the minimal anatomical disruption with the electrode implant procedure, the usual side effects of dysphagia, gas bloat or diarrhea seen with traditional antireflux surgery were not encountered in our patients [6]. An ongoing international multicenter trial has reported comparable results in a more diverse patient population and across multiple operators [20]. Thus far in our experience, the safety profile of LES stimulation appears to be quite superior to traditional antireflux surgery [6]. However, continuous monitoring of any safety events in a larger group of patients, treated across multiple practice settings over a longer follow-up period, is required to conclusively establish the safety of this therapy.

There are limitation of our study, primarily the small number of patients and open-label study design. We believe that the profound improvement in 24-h esophageal acid exposure sustained over a 3-year period is highly unlikely to be a placebo response [21]. Additionally, our blinded turn-off and turn-on sub-study points to a causal effect of LES stimulation on improvement in the esophageal acid exposure. A larger experience in diverse group of patients and operators is needed to validate our trial results for a wider application of this procedure. Such data are currently being collected in the international multicenter trial and in routine clinical practice in an international multicenter registry. A well-designed sham control trial would be helpful in overcoming the limitations of an openlabel design.

Comparative effectiveness study against standard antireflux surgery has been recently discussed [22, 23]. However, such a study is likely to be quite difficult, given 
issues of patient preference and generalizability of the results to less stringent patient population and operators. We believe that the LES stimulation therapy fills a "therapy gap" or an "under-met need" between pharmacotherapy and traditional antireflux surgery, particularly given its excellent safety profile. Additionally, due to the lack of any negative effect on esophageal body or LES motor function demonstrated on high-resolution manometry, LES stimulation therapy may be a desirable option in specific patient populations such as those with severe esophageal dysmotility including aperistalsis, post-myotomy GERD in patients with achalasia and in patients with GERD following laparoscopic sleeve gastrectomy for obesity. Early experience suggests that LES stimulation maybe effective in the latter group of patients.

In conclusion, at 3-year follow-up LES stimulation therapy effectively controls esophageal acid exposure and eliminates GERD symptoms and the need for regular PPI medications in majority of GERD patients that were at least partial PPI responder. LES stimulation had no long-term side effects and was associated with minimal adverse events mainly restricted to the post-op period. We believe that based on our long-term results and those being reported from the international multicenter trial [20], LES stimulation could be a therapeutic option for well-informed and select patients with GERD who are seeking an alternative to current medical or surgical therapies for GERD.

\section{Compliance with ethical standards}

Disclosures Study Sponsor-EndoStim BV, The Hague, Netherlands, provided funding for the trial. Prof. Michael D Crowell is a consultant for EndoStim Inc. and the chair of its data monitoring committee. Prof. Edy Soffer is a stock holder in EndoStim Inc. Leonardo Rodríguez, Patricia A Rodriguez, Beatrice Gómez, Manoel Galvao Netto, or their immediate family have no financial relationship with the sponsor that would constitute a conflict of interest.

Open Access This article is distributed under the terms of the Creative Commons Attribution 4.0 International License (http://crea tivecommons.org/licenses/by/4.0/), which permits unrestricted use, distribution, and reproduction in any medium, provided you give appropriate credit to the original author(s) and the source, provide a link to the Creative Commons license, and indicate if changes were made.

\section{References}

1. El-Serag HB, Sweet S, Winchester CC, Dent J (2014) Update on the epidemiology of gastro-oesophageal reflux disease: a systematic review. Gut 63:871-880

2. Wiklund I (2004) Review of the quality of life and burden of illness in gastroesophageal reflux disease. Dig Dis 22:108-114

3. Lagergren J, Bergström R, Lindgren A, Nyrén O (1999) Symptomatic gastroesophageal reflux as a risk factor for esophageal adenocarcinoma. N Engl J Med 340:825-831
4. Jamieson JR, Stein HJ, DeMeester TR, Bonavina L, Schwizer W, Hinder RA, Albertucci M (1992) Ambulatory 24-h esophageal pH monitoring: normal values, optimal thresholds, specificity, sensitivity, and reproducibility. Am J Gastroenterol 87:1102-1111

5. Fass R, Shapiro M, Dekel R, Sewell J (2005) Systematic review: proton-pump inhibitor failure in gastro-oesophageal reflux disease-where next? Aliment Pharmacol Ther 22:79-94

6. Richter JE (2013) Gastroesophageal reflux disease treatment: side effects and complications of fundoplication. Clin Gastroenterol Hepatol 11:465-471

7. Spechler SJ, Lee E, Ahnen D, Goyal RK, Hirano I, Ramirez F, Raufman JP, Sampliner R, Schnell T, Sontag S, Vlahcevic ZR, Young R, Williford W (2001) Long-term outcome of medical and surgical therapies for gastroesophageal reflux disease: follow-up of a randomized controlled trial. JAMA 285:2331-2338

8. Vakil N, Shaw M, Kirby R (2003) Clinical effectiveness of laparoscopic fundoplication in a US community. Am J Med 114:1-5

9. Thibault R, Coron E, Sébille V, Sacher-Huvelin S, Bruley des Varannes S, Gournay J, Galmiche JP (2006) Antireflux surgery for non-erosive and erosive reflux disease in community practice. Aliment Pharmacol Ther 24:621-632

10. Wang YR, Dempsey DT, Richter JE (2011) Trends and perioperative outcomes of inpatient antireflux surgery in the United States, 1993-2006. Dis Esophagus 24:215-223

11. Kahrilas PJ (2008) Magnetic enhancement of the lower esophageal sphincter. Gastrointest Endosc 67:295-296

12. Rodríguez L, Rodriguez P, Gómez B, Ayala JC, Oxenberg D, Perez-Castilla A, Netto MG, Soffer E, Boscardin WJ, Crowell MD (2015) Two-year results of intermittent electrical stimulation of the lower esophageal sphincter treatment of gastroesophageal reflux disease. Surgery 157:556-567

13. Rodriguez L, Rodriguez P, G omez B, Ayala JC, Oksenberg D, Perez-Castilla A et al (2013) Long-term results of electrical stimulation of the lower esophageal sphincter for the treatment of gastroesophageal reflux disease. Endoscopy 45:595-604

14. Stefanidis D, Hope WW, Kohn GP et al (2010) Guidelines for surgical treatment of gastroesophageal reflux disease. Surg Endosc 24:2647-2669

15. Katzka DA, Paoletti V, Leite L, Castell DO (1996) Prolonged ambulatory $\mathrm{pH}$ monitoring in patients with persistent gastroesophageal reflux disease symptoms: testing while on therapy identifies the need for more aggressive anti-reflux therapy. Am J Gastroenterol 91:2110-2113

16. Charbel S, Khandwala F, Vaezi MF (2005) The role of esophageal $\mathrm{pH}$ monitoring in symptomatic patients on PPI therapy. Am J Gastroenterol 100:283-289

17. Milkes D, Gerson LB, Triadafilopoulos G (2004) Complete elimination of reflux symptoms does not guarantee normalization of intraesophageal and intragastric $\mathrm{pH}$ in patients with gastroesophageal reflux disease (GERD). Am J Gastroenterol 99:991-996

18. Shah NH, LePendu P, Bauer-Mehren A, Ghebremariam YT, Iyer SV, Marcus J, Nead KT, Cooke JP, Leeper NJ (2015) Proton pump inhibitor usage and the risk of myocardial infarction in the general population. PLoS One 10:e0124653

19. Yang YX, Lewis JD, Epstein S, Metz DC (2006) Long-term proton pump inhibitor therapy and risk of hip fracture. JAMA 296:2947-2953

20. Kappelle WFW, Bredenoord AJ, Conchillo JM et al (2015) Electrical stimulation therapy of the lower esophageal sphincter for refractory gastro-esophageal reflux disease-interim results of an international multicenter trial. Aliment Pharmacol Ther $42: 614-625$ 
21. Rothstein RI (2008) Endoscopic therapy of gastroesophageal reflux disease outcomes of the randomized-controlled trials done to date. J Clin Gastroenterol 42:594-602

22. Attwood SE (2015) Electrical stimulation for gastroesophageal reflux disease: formal randomized clinical trials are needed. Surgery $157: 568-569$
23. DeMeester TR (2015) Letter to the editor regarding the article: two-year results of intermittent electrical stimulation of the lower esophageal sphincter treatment of gastroesophageal reflux disease. Surgery. 2015 April 22. pii: S0039-6060(15)00197-X. doi: 10.1016/j.surg.2015.03.011. [Epub ahead of print] 